$\underline{\text { Review Article }}$

\title{
LIQUISOLID COMPACTS: AN INNOVATIVE APPROACH FOR DISSOLUTION ENHANCEMENT
}

\author{
ANJANA ANIL, LITHA THOMAS, PREETHI SUDHEER* \\ Department of Pharmaceutics, Krupanidhi College of Pharmacy, Bengaluru 560035 \\ Email: preetisudheer@gmail.com
}

Received: 22 Nov 2017 Revised and Accepted: 08 May 2018

\begin{abstract}
The challenge faced by the majority of the pharmaceutical products is the poor solubility of the drug candidates which leads to low bioavailability Liquisolid compact is one of the emerging techniques that enhances the dissolution of poorly water soluble drugs. Liquisolid system mentions to the formulation made by the transforming the liquid drug, either in the form of suspension or solution in non volatile solvents into a dry, non-sticky, free-flowing and compactable powder mixtures. This is achieved by mixing the suspension or solution of the drug with appropriate carriers and coating agents. The technology has the ability to increase aqueous solubility, rate of dissolution and absorption of poorly soluble drug by keeping it in molecularly dispersed form leading to its improved bioavailability when compared to conventional tablets. Liquisolid technology is the impending approach for enhancing the solubility of poorly water-soluble drug by adopting simple manufacturing process and low production cost.
\end{abstract}

Keywords: Bioavailability, Liquisolids, Compressibility, Carrier, Coating agent, Loading factor, Solubility

(C) 2018 The Authors. Published by Innovare Academic Sciences Pvt Ltd. This is an open access article under the CC BY license (http://creativecommons.org/licenses/by/4.0/) DOI: http://dx.doi.org/10.22159/ijpps.2018v10i6.25500

\section{INTRODUCTION}

Drugs belonging to biopharmaceutics classification system (BCS) class II encounter the problem of low solubility, dissolution rate which leads to poor bioavailability [1]. Micronization, lyophilization, solid dispersion, co-solvancy and complexing agents are the common methods employed to enhance the dissolution rate of BCS class II drugs. However, the liquisolid compacts are one of the successful procedures that improve the bioavailability of these drugs [2]. "Liquisolid technology" also referred to as "powder solution technology". In this technology, a formulation is prepared by converting liquid drugs i. e solution or suspension from of drug in nonvolatile solvents into a dry non-sticky, compressible, smoothly flowing powder. This is achieved by mixing the drug suspension or solution with designated carriers and coating agents [3].

The dissolution profile of the drugs was found to increase from liquisolid method due to the increased wetting properties and surface area. The technique is limited to low dose drugs with poor water solubility [4]. The carrier used in liquisolid compacts should have high adsorption properties and have a porous surface towards the liquid medication. The commonly used carrier in liquisolid compacts is microcrystalline cellulose (avicel PH 20, 102, 200) [5]. Coating material (E. g. silica) is used to cover the surface, to impart flowability to the powder and to provide content uniformity to the manufactured tablet dosage form. The rate of release of the drug, water solubility, and wettability can be increased by the use of disintegrants. (Eg; starch glycolate and crosspovidone). Nonvolatile solvents must be water miscible and should be able to impart binding action to the product (polyethene glycol 200,400 and glycerin) [6].

\section{Advantages}

- The liquid-solid systems are versatile in the fact that it can be used for poorly soluble drugs.

- Improves the bioavailability of water-insoluble drug candidates, which are given by oral route (E. g. Risperidone, Griseofulvin, Carvidelol).

- When compared to soft gelatin capsules, the manufacturing expenditure is low.

- The drug can be formulated as a tablet or a capsule or as an encapsulated liquisolid microsystem, where the drug is presented in solubilized state which leads to enhanced wetting phenomena and improvement in drug release profiles.

- Instant release or continual release dosage forms can be formulated into liquisolid compact depending on the character of carriers used.

- Drug release can be improved by using suitable formulation excipients such as hydrophobic carriers (Eudragit RL) for sustained release, use of surface active agents (polysorbate 80) for improved wettability and hence enhanced dissolution profile can be accomplished.

- The technique can be scaled up to manufacturability.

- When compared to conventional tablets the extent of absorption can be enhanced up to $15 \%$.

- The manufacturing efficiency can be improved [7-9].

\section{Limitations}

- This methodology cannot be used to prepare high dose waterinsoluble drugs.

- It is observed that there is an increase in weight of tablet due to presence of carrier material and coating materials in larger levels.

- Application of mathematical calculations are required.

- Faster drug release can be achieved by ingredients with high absorption capacity which provide smaller tablet size.

- The inadequate hardness of liquisolid tablets results when acceptable compression is not achieved.

- Dissolution rate and bioavailability depends on the solubility of drugs in non-volatile liquids [10-12].

\section{Theoretical facts in the liquisolid system}

Only to a limited quantity of the liquid can be added above which the powder will lose its ability to retain the required flow and its compression properties.

The mathematical approaches, as mentioned by Spireas include two decisive powder characteristics, $\Phi$-value, which estimates the maximum liquid retention potential of powder with acceptable flow behavior. By measuring the rate of flow or repose angle, the 
flowability can be calculated. The $\Psi$-number this parameter gives an indication about compressible nature of powder with an adequate hardness along with maximum liquid retention potential. The compatibility determies the maximum crushing strength of unit weight of tablets.

Another important parameter is excipients ratio (ER), which represents the weight of carrier (C) to the coating agent (CA) present in the formulation:

$$
E R=C / C A
$$

The ER values of the powder substrate depends maximum liquid on carrier. An excipient ratio which can result in a liquid system with adequate flowability and compressibility, only the conditions where the liquid load on the carrier material is not over exceeded. This ratio is designated as "liquid load factor" LLf $[\mathrm{w} / \mathrm{w}]$ is the weight of the liquid formulation (W) to the carrier material (C) in the system:

$$
L L f=W / C
$$

The (LLf) guarantees adequate flow behavior ( $\Phi L L f)$ and is determined by:

$$
\phi L L f=\Phi+\psi \cdot(1 / E R)
$$

In the same way, the liquid load factor for liquisolid systems with satisfactory compatibility (YLf) can be determined by:

$$
\Psi L L f=\Psi+\Psi \cdot(1 / E R)
$$

Therefore, the optimum liquid load factor (LLo) necessary for obtaining most favourable flow behavior and compressibility of the system is either QLLf or YLLf, whichever is lesser in magnitude.

Once the optimum LLF is calculated, accurate amounts of the carrier (C) and coating agent (CA) necessary for conversion of the particular quantity of liquid formulation (W) into an almost flowing and compactable liquisolid system may be calculated in the following manner $[13,14]$ :

$$
\begin{aligned}
A C & =W / L L O \\
A C & =A C / E R
\end{aligned}
$$

\section{Mechanism involved in improved drug release profile from} liquisolid systems

Various literature reports emphasis that, the liquisolid system has been used to improve release rate of weakly soluble and low dose drugs. The incorporation of high dose water-insoluble drugs can be achieved by additives polyvinyl pyrrolidine (PVP), hydroxyl propyl methyl cellulose (HPMC), polyethene glycol (PEG) 35000 [15]. These additives can increase the liquid absorption capacity of carrier and coating material [16]. The responsible mechanism for enhanced drug release is given below:

\section{Augmented surface area}

The increase in the surface area of the drug in a liquisolid system (fig. 1) results from a complete dissolution of the drug in a liquid vehicle which represents the drug insolubilized and molecularly dispersed state [16].

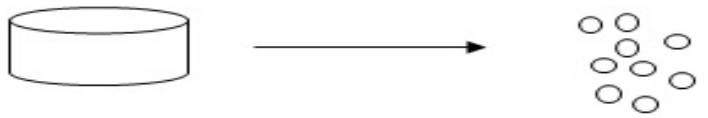

Fig. 1: Increased drug surface area [19]

A comparison between directly compressed tablets and tablets prepared liquisolid technique suggested that the drug release from latter was at a higher rate. The molecularly dispersed fraction of drug in liquid formulation is given by solubility of the drug divided by drug concentration which is expressed by the following equations [17]:

$$
F m=S / C
$$

Where,

$$
F m=1 S \geq C
$$

Fig. 2 represents the relation between molecularly dispersed states of the hydrocortisone from liquisolid compacts when propylene glycol (PG) was used as liquid media.

\section{Improved aqueous solubility of the drug}

Enhancement in the water solubility of the drug is due to the presence of a solid-liquid boundary between liquisolid primary particles and the media surrounding it. The quantity of the vehicle diffuses along with drug particle from the micro environmental condition contributes for an increase in the aqueous solubility (fig. 3), may be due to the cosolvent effect of the vehicle [20]

\section{Improved wetting properties}

The liquid vehicle has surface active properties which reduce the surface tension, results in the wetting of primary particles. The wettability can be determined by contact angle measurements (fig. 4) and the water rising time [21].

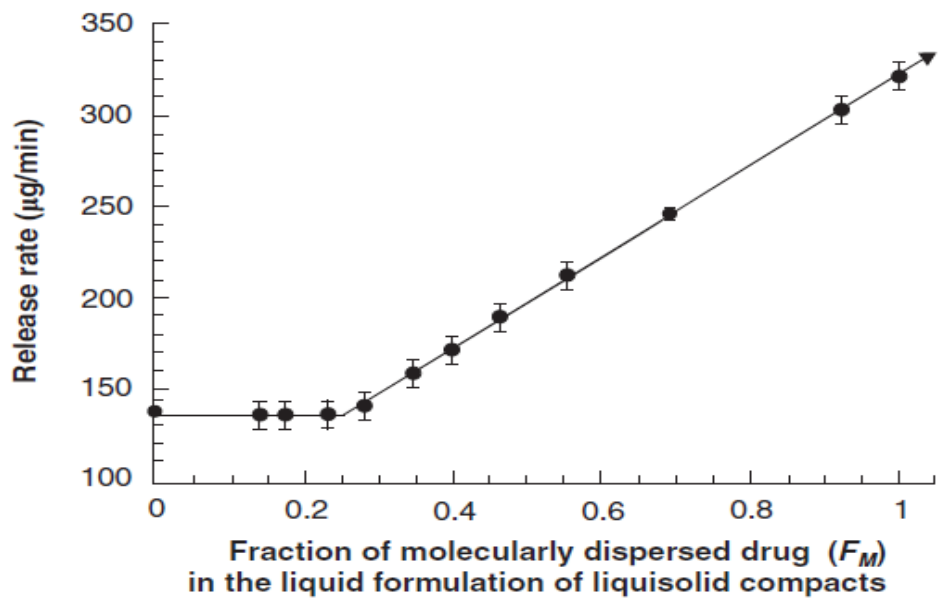

Fig. 2: Effect on the release rate of hydrocortisone liquisolid compacts 


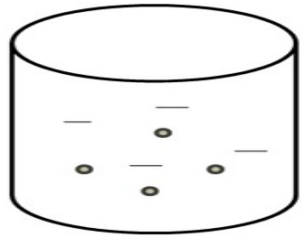

Dispersion

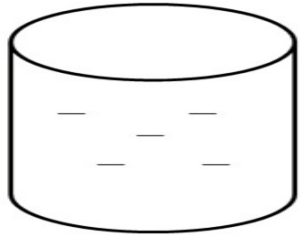

Clear solution

Fig. 3: Increased aqueous solubility of the drug [19]

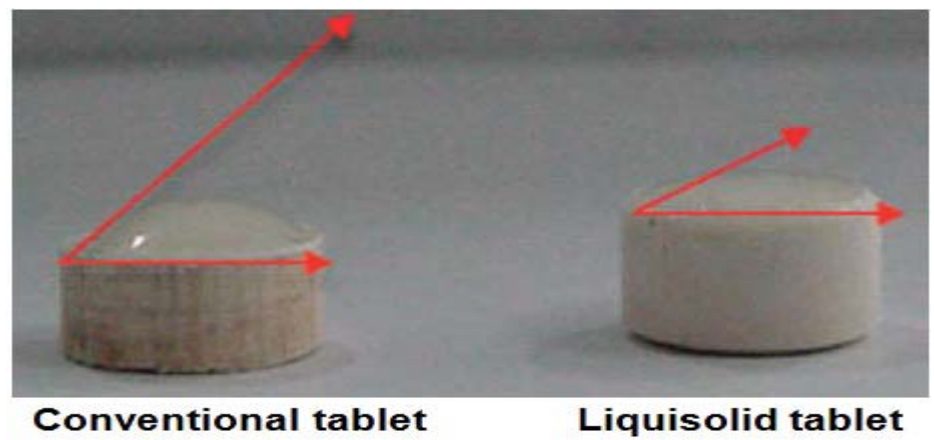

Fig. 4: Depiction of the contact angle of the conventional and liquisolid tablet [18]

\section{Porosity}

Pezzini et al. reported that liquisolid technology leads to soft structures with a high porosity which enhances the disintegration and dissolution process of liquisolid compacts of felodipine pellets [22].

\section{Mechanism of sustained drug release from liquisolid systems}

The replacement of the usual hydrophilic carrier with the hydrophobic carrier can lead to poor wetting which results in slow disintegration and thus extends the release of the drug. It is observed that there is neither a change in crystalline nature of drug nor a complex formation takes place during the process of liquidsolid compacts. This observation is much confirmed by X-ray crystallography and DSC measurements [23]. Another reason for decreased drug release is the influence of liquid vehicle, which was evident from the comparative study of directly compressed matrix tablets prepared of Eudragit ${ }^{\circledR}$ RS or RL as matrix forming material and liquisolid compacts, contains polysorbate 80 as liquid vehicle. Further, retardation was observed to a higher extent for the release of the drug from the liquisolid compact in contrast to conventional matrix tablets [24]. The coalescence observed with polymer particles in liquisolid compacts is much lower than with traditional matrix tablets. The reason for decreased drug release is due to the distinct coalescence of polymer particles, leads to decreased porosity and increased tortuosity [25]. The hydrophobicity of the polymer may contribute to the sustained release of the drug. The use of HPMC in the hydrophilic matrix system retards the drug-retaining by undergoing molecular weight dependent swelling. This system, when contact with water either swells or erodes results in zero order kinetics [27]. It is reported that the drug retardation effect from HPMC liquisolid compacts was more evident in comparison to directly compressed tablets [28].

\section{Formulation of liquisolid compacts}

Liquisolid compacts formula contains a carrier, coating material, nonvolatile solvent, disintegrants, lubricants and binding agents (table 1).

\section{Carrier material}

The carrier used should be spongy in nature, should posses satisfactory absorption properties for a liquid vehicle, both carrier as well as coating materials should hold a limited quantity of liquid, at the same time it should maintain flowability and compressibility. E. g, microcrystalline cellulose (MCC) (avicel PH 200 and avicel PH 102) [33].

\section{Coating material}

Coating materials are usually coarsely powdered particles which provide covering to the particles that are wet by adsorbing the excess of liquid, results in a dry free-flowing powder. E. g:-Various grades of silica (Syloid 244FP, Cab-O-Sil M5 and Aerosil 200) [34].

\section{Nonvolatile solvent}

Solvents used are nonvolatile, water-miscible, inert and not extremely viscous. They should have a high boiling point, possess good solubilization power for drugs used. Binding action can also be provided within the formulation with the help of nonvolatile liquids. E. g:-glycerin, polysorbate 80, propylene glycol, polyethylene glycol 200 and 400 [35].

\section{Disintegrating agents (disintegrants)}

These are agents which take up water, increases wettability, water solubility and the rate of drug release. The breakup of compacts into smaller particles can be achieved by the use of disintegrants. E. g sodium starch glycolate and cross-povidone, explotab and pregelatinized starch [36].

\section{Drug candidate}

BCS class II and IV drugs are generally choosen as a drug candidate for the liquisolid system. This results in increased water solubility of such candidates. E. g.-Naproxen, Digitoxin, Prednisolone, Hydrocortisone, Ketoprofen [37].

\section{Methodology}

The required quantities of the drug and stated quantity of nonvolatile solvent is weighed, mixed and heated (if required) results in a solution of the drug. Carrier and coating materials is incorporated into the drug solution. The process of mixing is to be done as three stages as recommended by Spireas et al. (fig. 5) [39].

\section{First stage}

The weighed ingredients are to be combined at an estimated mixing rate of one rotation/second/minute, which will aid the liquid medication to contribute its role in the powder [40].

\section{Second stage}

The above mixture should be spread evenly on a mortar surface for about 5 min. This results in complete absorption of drug solution into the voids of powder particles [41]. 


\section{Third stage}

The above blend is to be mixed with a super disintegrant for $30 \mathrm{sec}$ at a blending speed, which will results in the final blend ready for compression [42].

\section{Preformulation studies}

Preformulation studies performed to confirm the physiochemical characterization and it includes the following studies [44]

- Solubility studies of the drug in solvents

- Sliding angle determination
- Flowable liquid retention potential

- liquid load factor (LLf)

- Liquid-solid compressibility test (LSC)

The solubility of drug in non-volatile solvents

A saturated solution of the drug is prepared and is used for solubility studies. A surplus of the drug is added to vehicles which results in saturated solution by employing the shaker for the solution at a given period of time under steady vibration. The filtrate of the drug solutions are then analyzed spectrophotometrically.

Table 1: Components and physical characteristics of the Liquisolid technique

\begin{tabular}{|c|c|c|c|c|c|}
\hline Components & Drug candidates & Nonvolatile solvent & Carrier materials & Coating materials & Reference \\
\hline Properties & $\begin{array}{l}\text { Low dose with BCS class II } \\
\text { and IV }\end{array}$ & $\begin{array}{l}\text { Water-miscible ability to solubilise } \\
\text { the drug, act as a binding agent }\end{array}$ & $\begin{array}{l}\text { Porous, absorptive } \\
\text { properties }\end{array}$ & $\begin{array}{l}\text { Fine, highly } \\
\text { absorptive particles }\end{array}$ & 38 \\
\hline Examples & $\begin{array}{l}\text { Carbamazepine, } \\
\text { Indomethacin, Prednisolone }\end{array}$ & PEG 200, PEG 400, Glycerin & $\begin{array}{l}\text { Eudragit RL, } \\
\text { Eudragit RS }\end{array}$ & $\begin{array}{l}\text { Aerosil 20030, } \\
\text { Syloid 244FP }\end{array}$ & \\
\hline
\end{tabular}

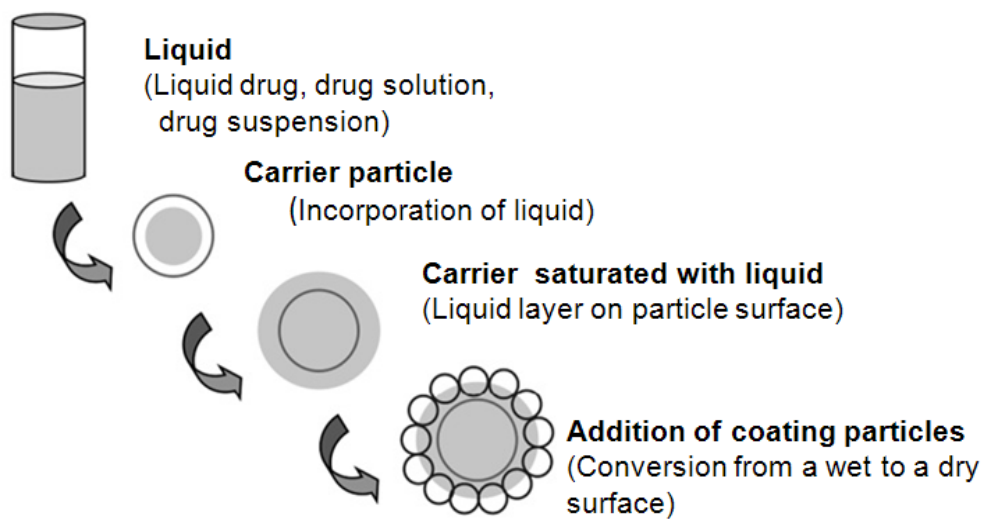

Fig. 5: Schematic representation of liquisolid preparation [43]

\section{Angle of slide}

Sliding angle measures the flow behavior of powders. A metallic plate with a smooth surface is used for the test, where the test powder is placed at one end of it, is gradually raised till the plate becomes angular to the horizontal plane, at which the powder just slides. The powder having an angle of $33^{\circ}$ provides optimum flow characteristics.

\section{Liquid flowable liquid retention potential ( $\Phi$ )}

It shows liquid retention potential of the powder with adequate flow behavior.

\section{Liquid load factor (LLf)}

It is the ratio of the weight of liquid medication (W) to the weight of carrier material (C). This is determined by taking adequate quantities of nonvolatile solvents in which the drug is dissolved, resulting solution is converted to a free flowing powder by addition of carrier and coating materials.

\section{Liquisolid compressibility test (LSC)}

This test determines the $\Psi$ values (compressible liquid retention value). It is done by preparation of carrier and coating material admixture, converting the admixtures into tablets. The average rigidity is measured by the average liquid content of crushed tablets $[45,46]$.

\section{Evaluation of liquisolid system}

- Flow behaviour

- Differential Scanning Calorimetry (DSC)
- X-ray diffraction (XRD)

- Scanning Electron Microscopy (SEM)

- Dissolution testing

- In vivo evaluation

Flow behavior

Bulk density

Weighed quantities of the powder blend is transferred into a graduated measuring cylinder. The bulk volume $(\mathrm{Vb})$ of the weighted amount of the powder (W) is determined. Bulk density is given below:

$$
\text { Bulk density }=W / V b
$$

\section{Tapped density}

The weighed amount of powder mass is poured to a graduated measuring cylinder and tapped for a fixed number of times and the volume is determined (Vt). Tapped density can be given by,

$$
\text { Tapped density }=W / V t
$$

\section{Compressibility index}

Compressibility index is given by the following equation:

Compressibility index $=($ tapped density - bulkdensity $/$ tappeddensity $) * 100$ 
Compressibility index values lower than $15 \%$ shows good flow characteristics of powders and values higher than $25 \%$ indicate poor flow nature.

\section{Hausner's ratio}

The indirect measurement of flow pattern of powders is given by:

$$
\text { Hausner's ratio }=\text { Tapped density } / \text { Bulk density }
$$

A value below $<1.25$ indicates good flow behavior, whereas $>1.5$ signify poor flowability. Hausner's ratio can vary depending on method used for the determination, so it is not taken as a critical parameter in flow behaviour. Flow property of powders are represented in the table 2

\section{Angle of repose}

The powder blend is passed through a funnel that is made to ascend vertically till the funnel tip touches the pile of the powder. The height of pile (h) and radius of the base of powder pile (r) is measured. The angle of repose is calculated as follows [48].

$$
\phi=\tan ^{-1} h / r
$$

Table 2: Flow behaviour of the powder

\begin{tabular}{llll}
\hline Compressibility index & Haussner's ratio & Flowability & Reference \\
\hline $5-15 \%$ & $1.05-1.18$ & Excellent & 47 \\
$12-16 \%$ & $1.14-1.19$ & Good & \\
$18-21 \%$ & $1.22-1.27$ & Fair-passable & \\
$23-35 \%$ & $1.30-1.54$ & Poor & \\
$33-38 \%$ & $1.49-1.69$ & Very poor & \\
$>40 \%$ & $>1.67$ & Very very poor & \\
\hline
\end{tabular}

\section{Differential scanning calorimetry (DSC)}

Thermal behavior of the pure components and the liquisolid compacts can be assessed by DSC studies. About 3-5 mg of the sample is vacuum-packed in aluminium pans exposed to the invariable rate of heating $10{ }^{\circ} \mathrm{C} / \mathrm{min}$ at a temperature range of 30 to $300^{\circ} \mathrm{C}$. Aluminum pans which are vacant are used as references and by purging nitrogen, the entire thermal behavior is studied. The absence of characteristic peak of the drug in presence of excipients is an indication of incompatibility of drug with excipients as well as changes in the crystalline pattern of the drug, may be a molecular level changes from a crystalline to amorphous pattern [49].

\section{X-ray diffraction (XRD) studies}

XRD studies determines the crystalline property of the liquisolid compact mixture by X-Ray diffractometer. The study uses a current of $30 \mathrm{~mA}$ and copper target at a voltage of $40 \mathrm{kV}$. The instrument works at a scanning angle of 5 to $70^{\circ}$ and a counting rate of 0.4 $\mathrm{s} / \mathrm{step}$. The change in the peak pattern from distinct and sharp to random pattern gives an evidence about the conversion of crystalline nature of drugs to amorphous forms of drug [50].

\section{Scanning electron microscopy}

This technique helps in determining the surface behavior of the drug, which gives an idea whether the drug is crystallized from the liquisolid system. The solubilized nature of the drug in liquisolid system results in the disappearance of these molecular forms [51].

\section{In vitro drug release studies}

In vitro release studies of the liquisolid tablets is performed using USP dissolution apparatus type II. The studies are carried out in 900 $\mathrm{ml} 0.1 \mathrm{~N} \mathrm{HCl}$ maintained at a constant temperature $37^{\circ} \mathrm{C} \pm 2{ }^{\circ} \mathrm{C}$ at a stirring speed of 50 to $200 \mathrm{rpm}$. After adding a known amount of drug equal formulation into the media, the percentage of drug dissolved is determined by withdrawing the samples at regular intervals and sink conditions are maintained by replacing with fresh buffer. The drug concentration can be determined spectrophotometrically (table 3 ) $[51,52]$.

Table 3: Conditions required for in vitro drug release studies

\begin{tabular}{ll}
\hline In vitro release parameters & Criteria \\
\hline Medium & Phosphate buffer $\mathrm{pH} 7.4$ \\
Apparatus & Basket type USP 24 (type II) \\
Medium volume & $900 \mathrm{ml}$ \\
Speed & $200 \mathrm{rpm}$ \\
Temperature & $37.0 \pm 0.5^{\circ} \mathrm{C}$ \\
Wave length & $282 \mathrm{~nm}$ \\
\hline
\end{tabular}

\section{In vivo evaluation of liquisolid tablets}

The comparative plasma concentration profile of the drug from the liquisolid compact in comparison to a commercial tablet should show a significant difference in area under plasma concentration profile, relative bioavailability, and peak plasma concentration [53].

\section{Applications}

Dissolution of drugs can be improved by liquisolid technique: Dissolution rate of low dose insoluble drugs such as Prednisolone [40], Famotidine [54], Valsartan [55] etc (table 4) can be enhanced by the use of the liquisolid technique.

Table 4: Liquisolid formula with improved drug release

\begin{tabular}{lll}
\hline Drug & Liquid vehicle & Carrier and coating material \\
\hline Aceclofenac & PEG 400 & MCC and HPMC \\
Famotidine & PG & MCC and Aerosil \\
Ibuprofen & PEG 300 & MCC and Aerosil \\
Prednisone & PG & MCC and Aerosil \\
Piroxicam & Polysorbate 80 & MCC and Aerosil \\
\hline
\end{tabular}


Incorporation of high dose water-insoluble drugs: by employing few additives such as PVP, HPMC and polyethylene glycol 35000, large dose, poorly soluble could be incorporated into liquisolid systems. This may be due to the fact that, these additives have the capability to increase the liquid uptake nature of carrier and coating materials and also by using modern carriers (such as
Neusilin ${ }^{\circledR}$ ) with augmented effective surface and greater absorption capacity [56].

Sustain drug release: Liquisolid technique a promising method for preparing the persistent release formulations of different drugs, as reported in the table 5 [57].

Table 5: Formulation of the liquisolid system with sustained drug release

\begin{tabular}{llll}
\hline Drug & Liquid vehicle & Carrier and coating material & Additional retardant agent \\
\hline Nifedipine & PEG 400 & MCC and colloidal silica & HPMC \\
Propranolol & Polysorbate 80 & Eudragit and colloidal silica & HPMC \\
Theophylline & Polysorbate 80 & Eudragit and colloidal silica & HPMC \\
Tramadol & PG & MCC and colloidal silica & HPMC \\
\hline
\end{tabular}

The control of $\mathrm{pH}$ deviation on drug release can be reduced by using liquisolid technology. When compared to marketed tablets and directly compressed tablets, the dissolution rate of liquisolid tablets is more and less affected by $\mathrm{pH}$ deviation on drug release [56].

Drug photostability in solid dosage forms can be enhanced by this method [56].

Liquisolid technology can be applied in probiotics [57].

\section{CONCLUSION}

Liquisolid technology is the impending move towards enhancing the solubility of the water-insoluble drug by using a simple industrialized process and lower production cost by the use of relatively inexpensive excipients. Due to the enhanced water solubility and dissolution rate, the extent of absorption of drugs can be increased. It can also be employed to design immediate and sustained release system by means of hydrophilic and hydrophobic carriers. Liquisolid technology employs liquid portion as suspensions or solution of poorly soluble drugs in a suitable nonvolatile liquid vehicle which are then changed to effortlessly smooth and compactable powders by simple physical blending with particular ingredients such as carrier and coating agent. This technology is found to be truly promising as the solubility and dissolution related problems of drugs, especially BCS class II and IV which leads to poor bioavailability can be surmounted as reported in the literatures.

\section{ACKNOWLEDGEMENT}

Authors express their sincere gratitude to The Management, Krupanidhi Group of institutions for supporting the work through research incubation centre (K-RIC) program under Krupanidhi College of Pharmacy and Dr. Parthasarathi, Accendere KMS, CL Educate Ltd.

\section{AUTHORS CONTRIBUTIONS}

All the author have contributed equally

\section{CONFLICT OF INTERESTS}

Authors have none of declare

\section{REFERENCES}

1. Vajir S, Sahu V, Ghuge N, Bakde BV. Liquisolid compact: a new technique for enhancement of drug dissolution. Int J Pharm Res Dev 2012;4:302-6.

2. Sowmya C, Reddy CS, Anilkumar D, Amrutha V, Anusha AL. Liquisolid technique: A novel approach to enhance solubility and bioavailability of BCS II drugs. Int Res J Pharm 2012;3:108-15.

3. Sanjay PD, Deepak M, Bhanudas SR. Liquisolid technology: a technique for formulation with enhanced bioavailability. World J Pharm Pharm Sci 2013;3:368-87.

4. Sanka Krishna, Sravanthi Poienti, Abdul Bari Mohd, Prakash V Diwan. Improved oral delivery of clonazepam through Liquisolid powder compact formulation: in vitro and ex vivo characterization. Powder Technol 2014;256:336-44.
5. Peddi MG. Novel drug delivery system: liquid-solid compact. J Mol Pharm Org Proc Res 2013;1:1-5.

6. Swati M. Solubility enhancement of a drug by liquisolid technique. Int J Pharm Chem Bio Sci 2014;4:339-44.

7. Sahir V, Ghuge N, Bakde BV. Liquisolid compact: a new technique for enhancement of drug dissolution. Int J Pharm Res Dev 2012;4:302-6.

8. Baby JN, Manjila SB, Bijen EN, Constantine I, Pramod K, Valsalakumari J. Design and technology of liquisolid compacts. J Appl Pharm Sci 2013;3:111-9.

9. Grover R, Spireas S, Wang T. Effect of powder substrate on the dissolution properties of methchrothiazide liquisolid compacts. Drug Dev Ind Pharm 1999;25:163-8.

10. Papadimitriou SA, Bikiaris D, Avgoustakis K. Microwaveinduced enhancement of the dissolution rate of poorly watersoluble tibolone from poly (ethylene glycol) solid dispersions. J Appl Polymer Sci 2008;108:1249-58.

11. Schiermeier S, Schmidt PC. Fast dispersible ibuprofen tablet. Eur J Pharm Sci 2002;15:295-305.

12. Tiong N, Elkordy AA. Effects of liquisolid formulations on the dissolution of naproxen. Eur J Pharm Biopharm 2009;73:373-84.

13. Nagabandi VK, Ramarao T, Jayaveera KN. Liquisolid Compacts. A novel approach to enhance bioavailability of poorly soluble drugs. Int J Pharm Bio Sci 2011;1:89-102.

14. Spireas S, Sadu S. Enhancement of prednisolone dissolution properties using liquisolid compacts. Int J Pharm 1998; 166:177-88.

15. Javadzadeh Y, Jafari-Navimipour B, Nokhodchi A, et al. a Liquisolid technique for dissolution rate enhancement of a high dose water-insoluble drug (carbamazepine). Int J Pharm 2007;341:26-34.

16. Hentzschel CM, Sakmann A, Leopold CS. Suitability of various excipients as carrier and coating materials for liquisolid compacts. Drug Dev Ind Pharm 2011;37:1200-7.

17. Hasanandini J, Parthibans S, Vilkeuwari A. Dissolution enhancement technique of poorly soluble drug by Liquisolid compact. Int J Res Pharm Nanol Sci 2014;3:298-304.

18. Nokhodchi A, Hentzschel CM, Leopold CS. Drug release from liquisolid systems: speed it up, slow it down. Expert Opinion Drug Delivery 2011;8:191-205.

19. Panda S, Varaprasad R, Priyanka K, Swain RP. Liquisolid technique: a novel approach for dosage form design. Int J Appl Pharm 2017;9:8-14.

20. Komala DR, Janga KY, Jukanti R. Competence of raloxifene hydrochloride loaded liquisolid compacts for improved dissolution and intestinal permeation. J Drug Delivery Sci Technol 2015;30:232-41.

21. Kumar Nagabandi V, Ramarao T, Jayaveera KN. Liquisolid compacts: a novel approach to enhance bioavailability of poorly soluble drugs. Int J Pharm Bio Sci 2011;1:89-102.

22. Pezzini BR, Beringhs AO, Ferraz HG. Liquisolid technology applied to pellets: evaluation of the feasibility and dissolution performance using felodipine as a model drug. Chem Eng Res 2016;110:62-9.

23. Khaled KA. Formulation and evaluation of hydrochlorothiazide liquisolid tablets. Saudi Pharm J 1998;6:39-46. 
24. Tayel SA, Soliman II, Louis D. Improvement of dissolution properties of carbamazepine through the application of the liquisolid tablet technique. Eur J Pharm Biopharm 2008; 69:342-7.

25. Rasenack N, Hartenhauer H, Muller BW. Microcrystals for dissolution rate enhancement of poorly water-soluble drugs. Int J Pharm 2003;254:137-45.

26. Azarmi S, Farid J, Nokhodchi A, Bahari-Saravi SM, Valizad H. Thermal treating as a tool for sustained release of indomethacin from Eudragit RS and RL matrices. Int J Pharm 2002;246:171-7.

27. Azarmi S, Farid D, Azodi-Deylami S, Ghaffari F, Nokhodchi A. The influence of Thermal treatment on the release behaviour of diclofenac sodium from an acrylic material. Pharm Dev Technol 2005;10:233-9.

28. Ghorab MM, Salam HM, El-Sayad MA. Tablet formulation containing meloxicam and $\beta$-cyclodextrin: mechanical characterization and bioavailability evaluation. AAPS Pharm SciTech 2004;5:1-6.

29. Gubbi S, R Jarag. Liquisolid technique for enhancement of dissolution properties of Bromhexine Hydrochloride. Res J Pharm Tech 2009;2:382-6.

30. Nokhodchi A, Y Javadzadeh, L Mosaalrezaei. Liquisolid technique for sustaining the drug release from compacts. J Pharm Res 2007;59:20-8.

31. Nokhodchi A. Liquisolid compacts: the effect of cosolvent and HPMC on theophylline release. Colloids Surf B 2010;79:262-9.

32. Gonjari ID, AB Karmarkar, AH Hosmani. Evaluation of in vitro dissolution profile comparison methods of sustained release tramadol hydrochloride liquisolid compact formulations with marketed sustained-release tablets. DJNB 2009;4:30-5.

33. Savkare AD, Bhavsar MR, Gholap VD, Kukkar PM. Liquisolid technique: a review. Int J Pharm Sci Res 2017;8:2768-75.

34. Kulkarni AS, Aloorkar NH, Mane MS, Gaja JB. Liquisolid systems: a review. Int J Pharm Sci Nanotech 2010;3:795-802.

35. Javadzadeh Y, Jafari-Navimipour B, Nokhodchi A. Liquisolid technique for dissolution rate enhancement of a high dose water-insoluble drug (carbamazepine). Int J Pharm 2007;341:26-34.

36. Sharma G, Khatry S, Arora S. Formulation and characterization of liquisolid compacts of valsartan. J Pharm Res 2012;5:4158-62.

37. Khan A, Agrawal S. Formulation and evaluation of lumefantrine capsule prepared by using the liquisolid technique. Int J Curr Pharm Res 2018:10:43-50.

38. Boghra R, Mahyavanshi T, Patel A, Sarode N, Jadhav N. Formulation and evaluation of Simvastatin liquisolid tablets. Asian J Pharm Sci Clin Res 2011:1:16-26.

39. Spireas S, Jarowski CI, Rohera BD. Powdered solution technology: principles and mechanism. Pharm Res 1992;9:1351-8.

40. Spireas S, Sadu S, Grover R. In vitro release evaluation of Hydrocortisone liquisolid tablets. J Pharma Sci 1998;87:867-72.

41. Hitesh Jain, Pasha TY, Bais CS, Anil Bhandari. Formulation and characterization of liquisolid tablets of Valsartan for improvement of dissolution rate. Asian J Pharm Clin Res 2014;7:21-6.

42. Saharan, Kukkar V, Kataria M, Gera M, Choudhury P. Dissolution enhancement of drugs. Part I: technologies and effect of carriers. Int J Health Res 2009;2:107-24.

43. Pawar JD, Jagtap RS, Doijad RC, Pol SV, Desai JR, Jadhav VV, et al. Liquisolid compacts: a promising approach for solubility enhancement. J Drug Delivery Ther 2017;7:6-11.

44. Stegemann S, Leveiller F. When poor solubility becomes an issue: From early stage to proof of concept. Eur J Pharm Sci 2016;31:249-56.

45. Gavhane KS, Sayyed F. Liquisolid compact a review. Int J Pharm Bio Res 2013;4:26-31.

46. Patel N. Liquisolid dosage system: a novel approach for dosage formulation. J Pharm Sci Biosci Res 2014;4:125-30.

47. Parthasarathi S, Anandharamakrishnan C. Enhancement of oral bioavailability of vitamin E by spray-freeze drying of whey protein microcapsules. Food Bioproducts Processing 2016;100:469-76.

48. Panda S, Varaprasad R, Priyanka K, Swain RP. Liquisolid technique: a novel approach for dosage form design. Int J Appl Pharm 2017:9:8-14.

49. Thakur N, Khokra S, Sharma D, Purohit R, Arya V. A review on pharmaceutical application of liquisolid echnique. Am J Pharma Tech Res 2011;1:1-18.

50. Sanjay PD, Deepak M, Bhanudas SR. Liquisolid technology: a technique for formulation with enhanced bioavailability. World J Pharm Pharm Sci 2013;2:368-81.

51. Khaled KA, Asiri YA, El-Sayed YM. In vivo evaluation of hydrochlorothiazide liquisolid tablet in beagles dogs. Int J Pharm 2001;222:1-6.

52. Rakshit P, Ridhish P, Moinuddin S. Formulation and evaluation of liquisolid compacts of piroxicam. Indian Drugs 2007;44:967-72.

53. Aher SB, Shinkar DM, Saudagar RB. Liquisolid dosage system: a novel approach for dosage formulation. Benefits Int J Pharm 2014;10:11-5.

54. Fahmy RH, Kassem MA. Enhancement of famotidine dissolution rate through liquisolid tablets formulation: in vitro and in vivo evaluation. Eur J Pharma Biopharm 2008;69:993-1003.

55. Chella N, Shastri N, Tadikonda RR. Use of the liquisolid compact technique for improvement of the dissolution rate of valsartan. Acta Pharma Sin B 2012;2:502-8.

56. Lu M, Xing H, Jiang J, Chen X, Yang T, Wang D, et al. Liquisolid technique and its application in pharmaceutics. Asian J Pharm Sci 2016;12:115-23.

57. Sander C, Holm P. Porous magnesium aluminometasilicate tablets as a carrier of a cyclosporine self-emulsifying formulation. AAPS Pharm Sci Tech 2009;10:1388-95.

58. Schlack H, Bauer brandel A, Schubert R, Becker D. Properties of fujicalin a new modified anhydrous dibasic calcium phosphate for direct compression: Comparison with dicalcium phosphate dihydrate. Drug Dev Ind Pharm 2001;27:789-801. 